\title{
Laboratory for Process Research - Ten Years of Successful Partnership between University of Zurich and the Pharmaceutical Industry
}

\author{
Thomas Bader
}

\begin{abstract}
In 1996, the Laboratory for Process Research (Labor für Prozessforschung = LPF) was founded as a collaboration between the University of Zurich and the pharmaceutical industry. This joint venture is based on the concept of providing a professional training platform in industrial process chemistry for Ph.D. graduates and performing highly sophisticated process research and development at the same time. The well-equipped LPF facilities, approved by Swissmedic, operate according to the standards of Good Manufacturing Practice (GMP). Chemical processes for manufacturing of active pharmaceutical ingredients (API) are developed from bench to production under GMP compliance. A large number of projects were successfully developed in the last decade, for which production was carried out at the LPF and/or after transfer of the whole technology to customers or contract manufacturers worldwide. In the course of these projects, the development of numerous innovative reactions and routes has resulted in several patents.
\end{abstract}

Keywords: API synthesis · GMP facility in university environment $\cdot$ Joint venture . Process research and development - Training in industrial process chemistry

\section{Introduction}

Cooperation between universities and industry can offer the industrial partner an opportunity to accelerate the time for research and development by working with highly skilled personnel experienced in different specific research disciplines, and by having access to academic resources like advanced and specific instrumentation. For their part, the academic researchers have the chance to gain insight into industrial practice, to improve their know-how with regard to practical application and to become more attractive job candidates because of their

\footnotetext{
${ }^{*}$ Correspondence: Dr. T. Bader

Labor für Prozessforschung

Organisch-chemisches Institut

Universität Zürich-Irchel

Winterthurerstrasse 190

$\mathrm{CH}-8057$ Zürich

Tel.: +4144635 4290

Fax: +4144635 3992

E-Mail: thomas.bader@|pf.unizh.ch
}

advanced practical knowledge and experience.

Thus in 1996 a collaboration of the University of Zurich (UZH) with the Swiss company Cilag AG (which is integrated in the Pharmaceuticals Group of Johnson \& Johnson) resulted in the foundation of the Laboratory for Process Research (LPF). From the university side a technical laboratory for industrial scale synthesis was provided, whose infrastructure was improved by Cilag to provide a facility able to perform GMP production for Cilag, and useful as a process chemistry training platform for UZH. In the following years, a large number of multi-step synthetic campaigns for intermediates and final compounds of active pharmaceutical ingredients were successfully executed, the latter mostly essential for the clinical trials at Johnson \& Johnson. Inspections by Swissmedic at regular intervals as well as customer audits, certified GMP compliance at the LPF.

In 2003, the UZH adopted full control of the lab and recruited Professor Jay S. Siegel as the Executive Director of the LPF. Shortly thereafter, a joint entrepreneurial venture was undertaken with the generic API firm AZAD, in which the LPF was responsible for the development of synthetic routes for the large-scale production of generic APIs, while AZAD retained control of all aspects of API marketing, sales and regulatory affairs.

\section{LPF not only a Joint Venture but also a Partner in Contract Research}

In addition to its work with AZAD, the LPF also has the possibility to pursue additional projects with 'third party' customers. To date, several projects for third party clients have been successfully developed. Importantly, both the contract partner and other clients can rely on professional expertise and service from an experienced international team, coupled with a strictly confidential handling of all proprietary information.

\subsection{Organization of the LPF and Field of Activities}

2.1.1. Overview and General Aspects

The Laboratory for Process Research comprises three divisions: Research \& Development, Analytics and Production. Two thirds of the international staff members working within these divisions hold a Ph.D. in chemistry. More than one third 
of them are employed as postdoctoral researchers performing project research and development under exactly the same economic and timeline pressures as anywhere in the industry. In order to ensure that the LPF is both a competent and reliable partner, as well a superior training platform for academic graduates in process chemistry, a well-defined but flexible organizational structure had to be created. Within this organization (Fig. 1), the lead of the LPF is assigned to the Executive Director, who is responsible for core business such as contract negotiations, approval of budgets and investments. To assist with strategic planning, as well as to handle day-to-day operations, the LPF Leadership Team consisting of the Heads of Divisions and the Chemical Advisor was formed. Specific aspects of day-to-day business include the identification and implementation of requirements of contract partners and clients as well as internal aspects like budget and investment planning, personnel education and development. As an important task for ensuring GMP compliance at the LPF, the Leadership Team is part of the Quality Assurance (QA) group together with the Executive Advisor as Head of QA. Finally all aspects of LPF business are discussed in periodic meetings with the Executive Director.

\subsubsection{LPF - R\&D Department, Process Chemistry in a University Environment}

The largest division of the LPF is the R\&D Department. This is the section in which the chemical process development takes place (Fig. 2) and where academic graduates are trained in process chemistry, directly instructed by experienced group leaders. The R\&D facility includes the infrastructure necessary for rapid and full development of chemical processes: basic equipment includes nine HPLCs, two GCs and a GC-MS. In addition, a state-of-the-art reaction calorimeter (Fig. 3) was recently purchased to ensure complete safety investigations during process development.

Furthermore, since the LPF is integrated in a university environment, it has full access to university infrastructure, e.g. to NMR, $\mathrm{X}$-ray diffraction and mass spectrometric facilities enabling comprehensive studies of compounds and structures. For identification of crystalline forms, a new automated $\mathrm{X}$-ray powder diffraction system (Fig. 4) is available, and synchrotron measurements carried out at the Paul Scherrer Institute located nearby in Villigen allow detailed polymorphism studies, even in complex mixtures such as formulated APIs.

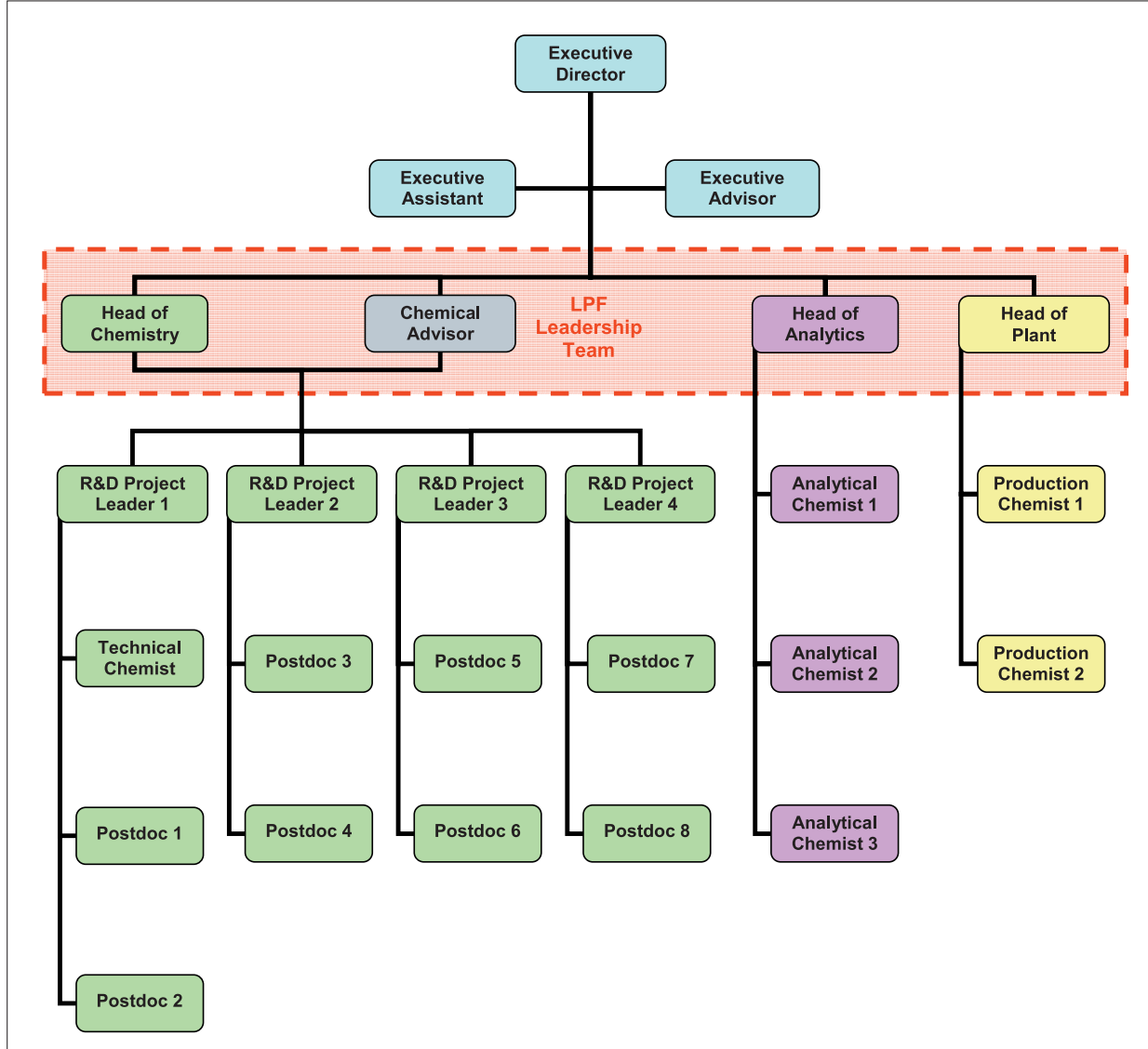

Fig. 1. Organization Chart of the LPF. Blue: Members of Directorate; green: Members of R\&D Department; violet: Members of Analytical Department; yellow: Members of Production Department; red: members of LPF Leadership Team.

$>$ Comprehensive patent and literature review

$>$ Design and development of competitive, non-infringing, innovative and patentable synthetic routes for small and complex molecules

Complete development of economic, robust, and scaleable synthetic processes including synthesis of impurities and reference standards

$>$ Process optimization of existing routes

$>$ Transfer of technology to custom/contract manufacturers

$>$ Cost calculations and estimation of timelines

$>$ Polymorph screening

Salt screening

Fig. 2. Overview of the R\&D activities at the LPF

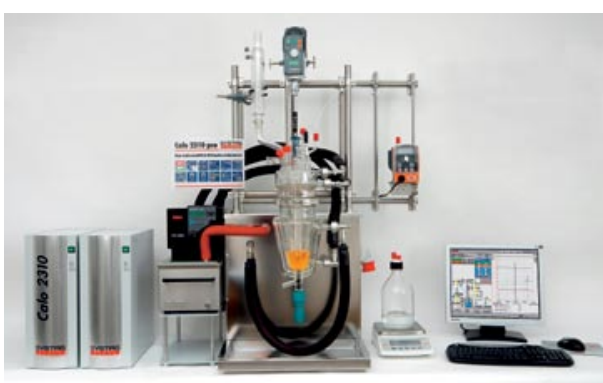

Fig. 3. Reaction Calorimeter 'Calo 2310 pro BS' for safety investigations

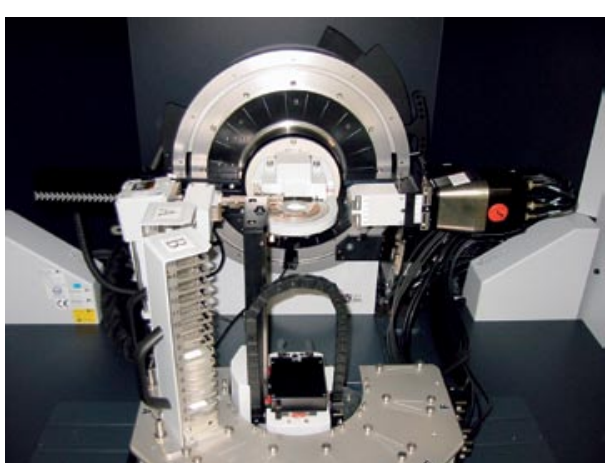

Fig. 4. D8 ADVANCE with VÅNTEC-1 Detector

\subsubsection{LPF - Analytical Department, a GMP Compliant Laboratory}

The LPF Analytical Department operates under full GMP compliance and as a QC Laboratory for the release of APIs. It provides the R\&D-Department, Produc- 
tion, customers and contract manufacturers with a full palette of analytical support even after completion of the project development at the LPF (Fig. 5).

A variety of state-of-the-art instruments are available: HPLC and GC instruments (analytical and 'chiral'), HPLC-MS (ion trap), FT-IR (ATR), DSC and headspaceGC (Fig. 6). Furthermore the spectrum of analytical determinations is completed by full in-house access to numerous high-field NMR instruments $\left({ }^{1} \mathrm{H},{ }^{13} \mathrm{C}\right.$ and other nuclei, 2D-NMR, solid state measurements), crystallography (XRD and single crystal X-ray analysis), Raman-IR, atomic absorption spectroscopy, polarimetry and circular dichroism, preparative HPLC and GC as well as external access to ICP-MS and synchrotron sources.

$\begin{aligned}> & \text { Method development for all types } \\ & \text { of HPLC and GC analysis } \\ > & \text { Full palette of EP/USP analysis } \\ > & \text { Purity and assay determination } \\ > & \text { Impurity profile characterization } \\ > & \text { Release of chemicals for GMP } \\ & \text { production } \\ > & \text { Release of final products (APIs) } \\ > & \text { Certificates of Analysis } \\ > & \text { Polymorphism studies } \\ > & \text { Long term stability studies } \\ > & \text { Structure elucidation } \\ > & \text { Thermochemical analysis } \\ > & \text { Karl Fischer titration for water } \\ > & \text { Combustion elemental analysis } \\ > & \text { Residue on ignition analysis } \\ > & \text { Residual solvent analysis }\end{aligned}$

Fig. 5. Overview of the analytical service at the LPF

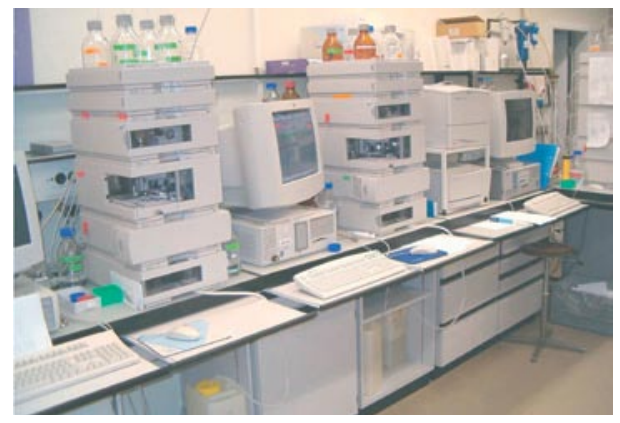

Fig. 6. Certified equipment in the Analytical Department

2.1.4. LPF - Production Department, a GMP Compliant Manufacturing Facility

The LPF production facility (Fig. 7,

Fig. 8) is designed for the GMP synthesis of pharmaceutical intermediates and final products for clinical trials up to phase III, in quantities from grams to kilograms, using a broad range of chemical reactions. A number of campaigns have been run through the production unit, and up to 16-step syntheses of complex molecules were successfully carried out in compliance with GMP.

$>6$ Reactors $(6-1001)$

$>4$ Stirring vessels $(50-2001)$

> 2 High capacity evaporators (20 1 per hour each)

$>$ Autoclave up to 10 bar (2-20 1)

$>$ Centrifuge

$>$ Powder handling unit with several ovens

Fig. 7. Overview of the equipment in the Production Department

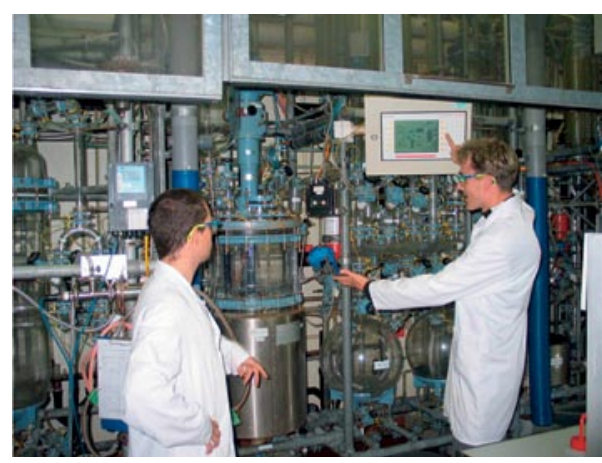

Fig. 8. LPF: Working at the 100 I reactor

Up to $10 \mathrm{~kg}$ product can be produced in a single batch. The operation conditions range from $-40{ }^{\circ} \mathrm{C}$ to $+160{ }^{\circ} \mathrm{C}$ in all reactors and from $-120{ }^{\circ} \mathrm{C}$ to $+220{ }^{\circ} \mathrm{C}$ in a 121 reactor. The plant is regularly inspected by Swissmedic as well as audited by clients.

\section{The LPF as a Training Platform in Process Chemistry for Postgraduate Chemists}

Process development at the LPF is focused primarily on route discovery and route optimization rather than drug discovery or identification of new lead compounds. This means that the final molecule is already known and that sometimes an existing route is available, but that a practical route has to be developed in order to provide the target compound in larger amounts. Of course, the aim is not only to find a suitable, scalable process but also to find one that is cost-efficient at commercial scale and that meets all the technical requirements necessary for large scale production of a high-quality product. The tasks for a process develop- ment chemist include considerations which are not routine in an academic research laboratory. Thus, when postdocs start their process chemistry training, they quickly realize that there is often a big difference between their previous academic work and the demands of process chemistry.

Generally, working in the field of process chemistry at the LPF includes extensive training according to internal SOPs and guidelines that have been formulated at the LPF during the last decade. Understanding the differences between carrying out a reaction in a lab scale or in pilot plant/production scale is essential to avoid failures during scale-up. Above all safety considerations are obligatory for the personnel and the environment, which means e.g. that the use of highly toxic or hazardous raw materials or reagents must be avoided and a formal literature or database research about available safety information (like material safety data sheets, MSDS) must be carried out. Detailed investigations of reaction thermodynamics (e.g. exothermic activity observed, is the reaction controlled by dosing of the reactants or is an accumulation observed?) are important to avoid runaway reactions. Furthermore, an understanding of the minimum criteria which have to be met, like reproducibility (the process must be reliable and robust), economically viable (cost and availability of raw materials, manufacturing costs which consist of e.g. the kind of equipment and time spend for reaction and work-up, volume yield etc.), environmental responsibility (waste avoidance, possibility for recycling of material etc.) and feasibility within the available plant (e.g. avoidance of cryogenic reactions that need expensive special equipment) is a precondition in process chemistry.

It is important to be aware that running a process on a larger scale means consideration of $e . g$. heat transfer, mixing effects and the processing time. Since heating or cooling of a mixture, distillation, phase separation, filtration, drying and the like take longer at scale, it is necessary to investigate the influence of these parameters already in the laboratory to avoid batch failure by $e . g$. decomposition of the product and formation of impurities. Therefore, the key parameters of the process must be identified, i.e. where is the uncritical range and which parameter are critical and whether there are sufficient tolerances for each process step for defining the operating conditions. The investigation must also include the characterization and identification of the impurity profile to obtain additional information about the mechanism of a reaction. These are key pieces of information for further optimization of the process by varying the conditions or reagents. At the end of an optimal developed route stands a thoroughly analyzed and defined process, wherein the scope and limita- 
tions of each step are known and specifications are set which fulfill the requirements for a large scale manufacturing to produce a high quality product. All results and information must finally become part of a welldocumented laboratory report as well as a status and/or development report.

These aspects are only some of the scale up issues investigated during process development, but unfortunately they are normally not part of the academic education of chemists. While a large volume of literature already address these issues (e.g. [1]) it generally takes a lot of time to acquire meaningful experience in this discipline. It is part of the mission of the LPF to fill the gap of experience for academic graduates, and to train chemists to consider the aspects of scale up. This training is done directly on projects from industrial partners or clients, which therefore have a real-life commercial context. Chemists leaving the LPF understand the requirements of scaling up, and they are well prepared for their future work in the field of process chemistry.

\section{Project Development at the LPF}

Within the last decade, a large number of projects were developed at the LPF. In particular, the development of generic APIs has become more important in the last years. Generally, analysis of market volume and potential, current patent situation, cost and timeline for the chemical development are among the important factors in the evaluation of new projects. Within this evaluation, the tasks of the LPF include the consideration of feasibility, current patent situation, design of alternative, non-infringing and innovative routes as well as protection of intellectual property, calculation of costs and timeline for the planned development and production and finally transfer of chemical technology and analytical methods to contract manufacturers or to customers. Many projects run through the LPF have led to technology transfer executed by LPF members within Europe and Asia. Products include not only final compounds but also reference standards, impurities, and the like, which have been provided to firms all over the world.

The following studies of two generic APIs (Anastrozole and Trandolapril) provide a representative overview of activities at the LPF during the evaluation and development of research projects. Demonstrated are consideration of patent and literature procedures, route discovery for more efficient and innovative processes, as well as polymorph screening and scale up.

\subsection{Anastrozole}

\subsubsection{Introduction and Background}

One of the projects worked on at the LPF was Anastrozole (brand name: Arimidex ${ }^{\circledR}$,
Fig. 9), chemical name 2,2'-[5-(1H-1,2,4triazol-1-ylmethyl)-1,3-phenylene]di(2methylpropionitrile) or $\alpha, \alpha, \alpha^{\prime}, \alpha^{\prime}$-tetramethyl-5-(1H-1,2,4-triazol-1-ylmethyl)1,3-benzenediacetonitrile, represented by formula A1.

Anastrozole is known to be a potent, selective and reversible non-steroidal aromatase inhibitor used for the treatment of advanced breast cancer in postmenopausal women [2].

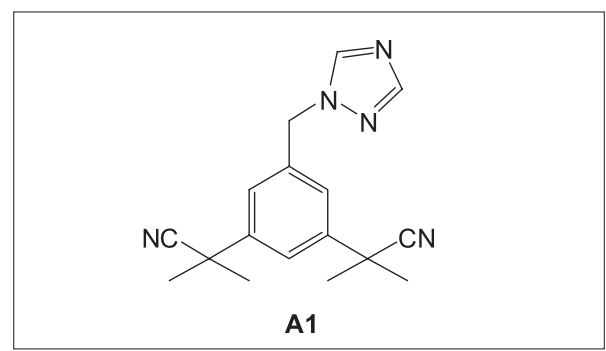

Fig. 9. Structure of Anastrozole

Two routes for the synthesis of Anastrozole are disclosed in [3] but without any specification of yields and purities. The first procedure (Scheme 1) starts with the conversion of compound $\mathbf{A 2}$ to $\mathbf{A} \mathbf{3}$ by a nucleophilic substitution of the benzylic bromides with potassium cyanide in the presence of catalytic amounts of tetrabutylammonium bromide in a mixture of dichloromethane and water. After isolation, the crude product was purified by flash chromatography, followed by crystallization from carbon tetrachloride.

The following step involves exhaustive $\alpha$-methylation of both nitrile groups in $\mathbf{A 3}$ to provide $\mathbf{A 4}$. The conversion was carried out in DMF in the presence of sodium hydride as the base and iodomethane as methylation reagent. Purification of $\mathbf{A 4}$ was conducted by crystallization from carbon tetrachloride. Radical bromination of the methyl group in A4 using N-bromosuccinimde and benzoyl peroxide as initiator gave compound $\mathbf{A 5}$, which was used directly as crude product for the next step. In the final step, A1 (Anastrozole) was prepared by substitution of benzyl bromide A5 with sodium triazole in DMF. The purification of the crude product was performed by flash column chromatography (eluent ethyl acetate) followed by crystallization from a mixture of ethyl acetate and cyclohexane.

The second process for preparing Anastrozole described in the aforementioned patent uses the readily available methyl 3,5-dimethylbenzoate A6 as starting material (Scheme 2).

After radical bromination of the methyl groups in A6, the resulting compound A7 is transformed to $\mathbf{A 9}$ in the same manner as previously described for A4. Compound A9 was then reduced with lithium borohydride to the benzyl alcohol A10, which is further converted to the corresponding benzyl chloride A11 using thionyl chloride. Finally, substitution of the benzylic chloride with $1 H$-1,2,4-triazole gave a crude product which is purified by flash column chromatography (eluent methanol/chloroform) to obtain Anastrozole A1 separated from its corresponding regio isomer A12 (Fig. 10)

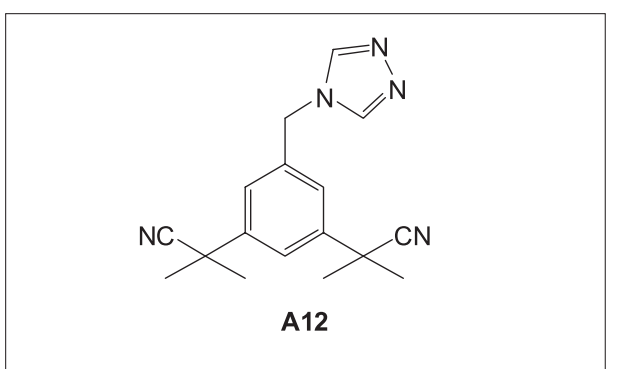

Fig. 10. Structure of the regio isomer of Anastrozole

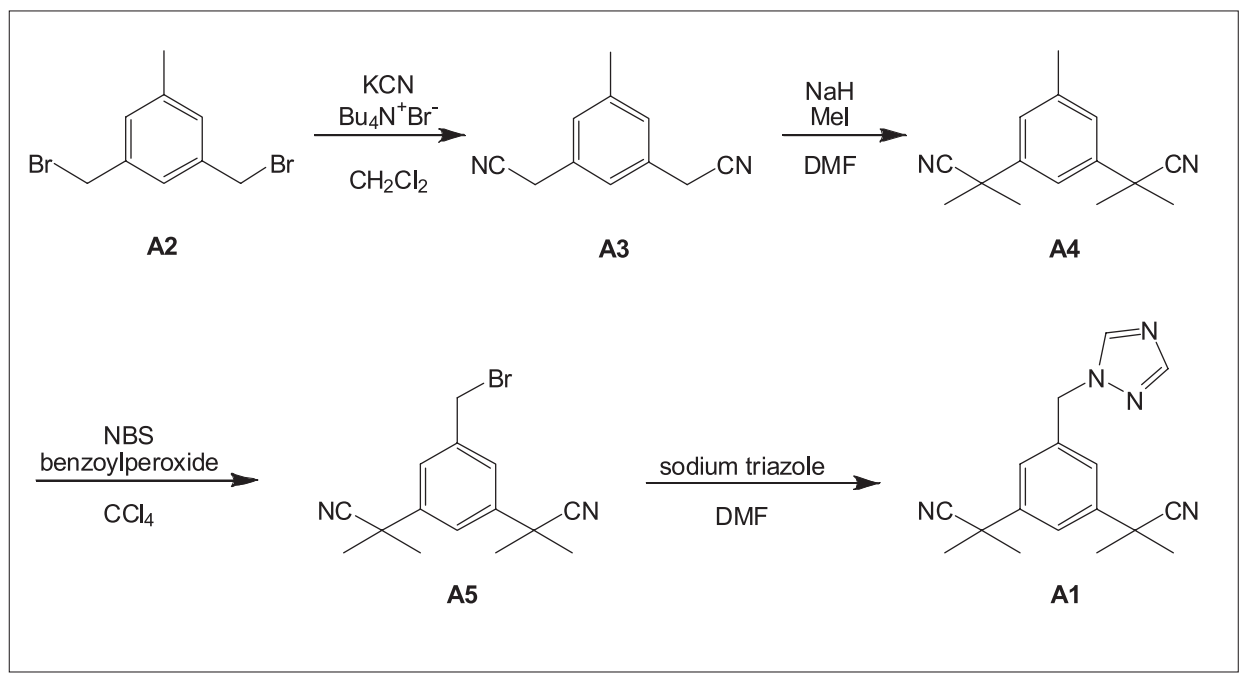

Scheme 1. First route by Edwards et al. for the synthesis of Anastrozole [3] 


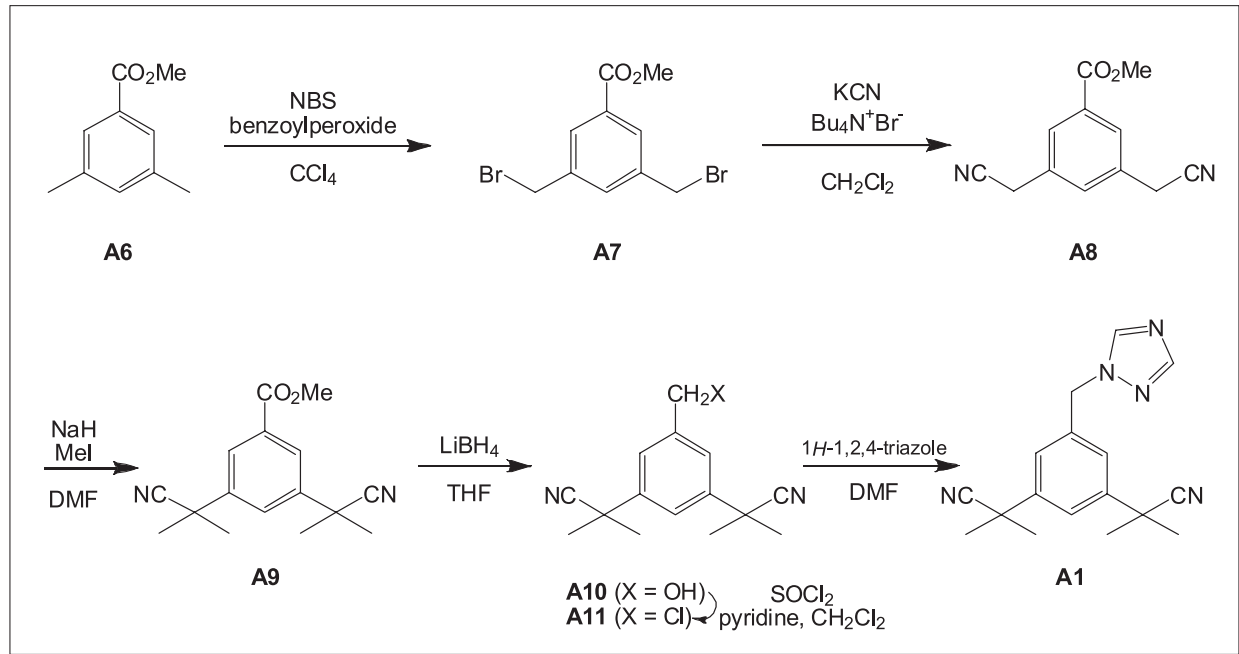

Scheme 2. Second route by Edwards et al. for the synthesis of Anastrozole [3]

formed as contamination product during the coupling step.

With respect to developing a feasible technical process, these strategies and methods for the preparation of Anastrozole have several serious drawbacks. First the compound $\mathbf{A} \mathbf{2}$ is not commercially available and has to be synthesized, e.g. from mesitylene by radical bromination. It is obvious that such reactions may form multiple products which have to be separated efficiently to obtain pure A2. Therefore a difficult purification procedure and loss of yield have to be taken into account. In a similar manner, the radical bromination of $\mathrm{A4}$ and $\mathrm{A6}$ also forms polybrominated compounds. As described by Deng and co-workers [4], polybrominated methyl groups in $\mathbf{A} 7$ can be reduced to the corresponding monosubstituted benzyl bromides using diethyl phosphite and N,Ndiisopropylethylamine, but this requires an additional step for the preparation of Anastrozole. Alternative preparation of the corresponding 3,5-bis-(bromomethyl)benzoic acid in four steps starting from 1,3,5-benzenetricarboxylic acid is described by Erickson and co-workers [5] but it has the disadvantage of lengthening the route dramatically.

Since carbon tetrachloride is known to be toxic and a suspected human carcinogen and mutagen, alternative solvents for the bromination reaction must be found. Furthermore, special precautions have to be considered for the substitution of the bromide with potassium cyanide as well as for the use of iodomethane because of their toxic properties. Additionally, the conversion of $\mathbf{A 3}$ and $\mathbf{A 8}$ into the corresponding tetramethyl derivatives A4 and A9 may cause the formation of difficult-to-separate byproducts by incomplete methylation. Moreover, although the use of sodium hydride in DMF as solvent is a procedure often described in the literature, chemists should be aware that exothermic and runaway reactions during scale up have been reported [6]. Finally, using column chromatography for purification of intermediates and the final compound is undesirable on production scale and less viable for commercial processes.

Recently several patents were published which overcome certain drawbacks of the aforementioned description. Two patents [7][8] disclose a procedure for purifying Anastrozole (A1) by formation and recrystallization of the corresponding salts to remove the impurity $\mathbf{A 1 2}$ or by extraction of this contaminant at a $\mathrm{pH}$ between 0.7 and 1.7 first followed by salt formation. Furthermore alternative solvents (e.g. methylene chloride, acetone, acetonitrile) for the bromination step to form $\mathbf{A 5}$ are also provided.

A new process for the preparation of Anastrozole is disclosed in [9] and shown in Scheme 3.
Scheme 3. Synthesis of Anastrozole by Gaitonide et al. [9]
The process provided in [9] starts with the conversion of $\mathbf{A 3}$ to $\mathbf{A 4}$ according to the known method as described in [3]. The next step involves an oxidation of the methyl group in A4 to the diacetate A13 using chromium trioxide, acetic anhydride and sulfuric acid. Hydrolysis of $\mathbf{A 1 3}$ gave A14, which is condensed with Boc-protected hydrazine resulting in the formation of A15. Reduction of the hydrazone A15 to the hydrazine derivative A16 followed by removal of the Boc-protecting group and salt formation with hydrogen chloride gave compound A17. The synthesis of Anastrozole was completed by reaction of $\mathbf{A 1 7}$ with s-triazine. Although the strategy selectively forms Anastrozole in an overall yield of $14.5 \%$ starting from A3 and in a HPLC purity of $>99.5 \%$, the use of chromium trioxide makes this method unattractive for production scale because of its toxic and carcinogenic properties and environmental impact. Furthermore, since seven steps starting from $\mathbf{A 3}$ are necessary for the preparation of Anastrozole, several having low volume yields (e.g. $<1 \%$ for the preparation of $\mathbf{A 1 3}$ ), this strategy seems to be commercially less attractive.

During our route development for an efficient process to produce pure Anastrozole in high yields it was our objective to find an alternative for the successive formation of the tertiary butyronitrile groups to overcome the problems of unselective bromination and incomplete methylation mentioned above. Furthermore the final coupling step, i.e. reaction of compound A5 with 1,2,4-triazole derivatives, was expected to give a mixture of A1 and A12. Thus in our first screening of the final coupling step selectivities between 88:12 and 94:6 for the compounds A1 and A12 were obtained by reacting compound

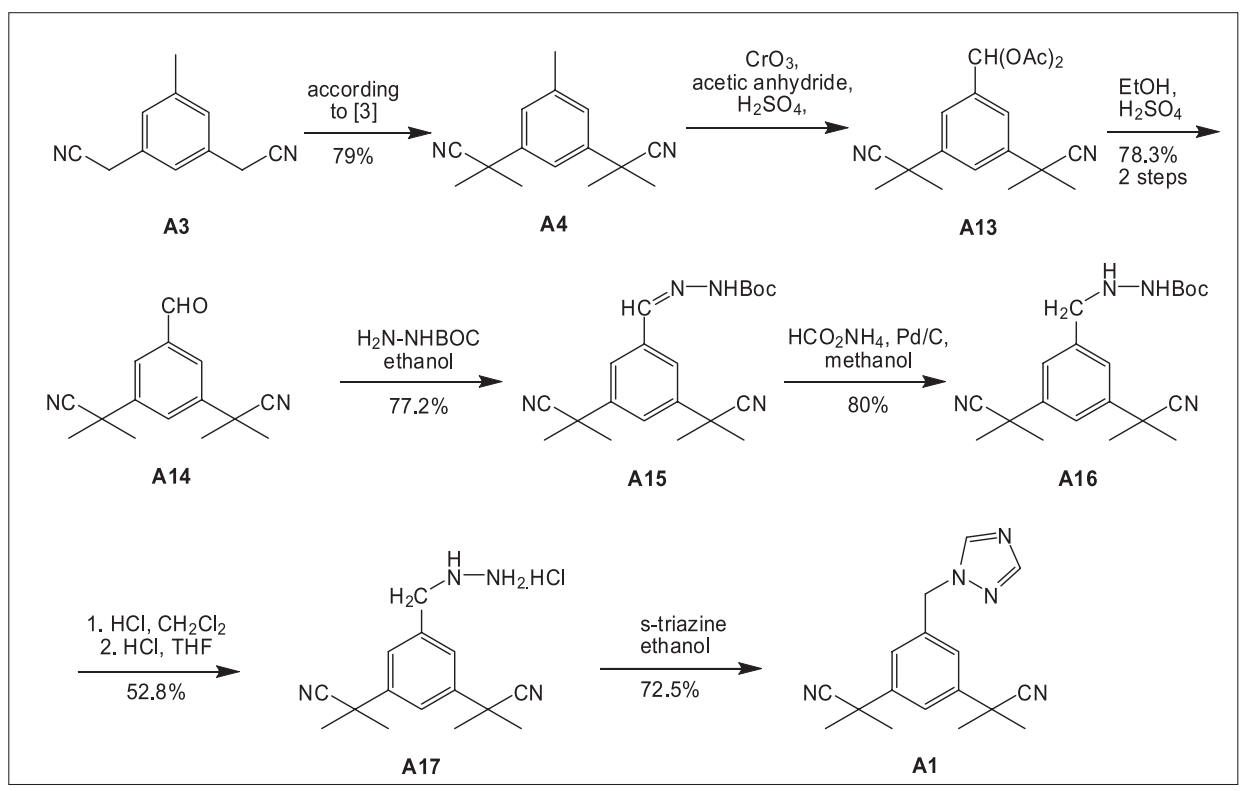


A5 with sodium triazole in dipolar aprotic solvents like dimethyl sulfoxide, dimethylformamide, $\mathrm{N}, \mathrm{N}$-dimethylacetamide or mixtures thereof with acetone. Although the amount of the undesired diastereomer was relatively low, removal of A12 by fractional crystallization or extraction always resulted in loss of yield and contamination with the undesired regioisomer. Therefore a more selective process for this step was also required.

\subsubsection{Development of a New Route}

During our route discovery, an alternative for the selective preparation of Anstrozole was found [10]. A general review of the literature about methods for building tertiary benzylic nitriles resulted in two interesting methods describing a formation in one step by palladium-catalyzed arylation of secondary nitriles using aryl bromides [11] or by nucleophilic aromatic substitution of aryl fluorides with secondary nitriles [12]. Since 3,5-difluoro-benzoic acid A18 is readily available, the latter method seemed to be suitable for further investigations (Scheme 4).

As described in the literature, the optimal conditions for the addition of secondary nitriles to fluoroarenes required 1.5 equiv. potassium hexamethyldisilazide (KHMDS) as the base, 4 equiv. of the nitrile and THF or toluene as the solvent. Although a variety of monofluorinated aromatic compounds bearing different substituents were investigated, the simultaneous substitution of two fluorides by secondary nitriles or the reaction with fluorinated benzoic acids were not described. Using the fluorinated benzoic acid $\mathbf{A 1 8}$ at least double amounts of the corresponding reagents were necessary. To avoid the waste of 1 equiv. KHMDS by deprotonation of the carboxylic acid, prior salt formation with a suitable cheaper base was considered. The best conditions for the conversion of A18 to A19 were the formation of the lithium salt of A18 using lithium hydride followed by substitution of both fluorides using 3.3 equiv. of KHMDS and 8.1 equiv. of isobutyronitrile. The reaction is typically carried out in $\mathrm{THF}$ at $70^{\circ} \mathrm{C}$. After recrystallization, compound $\mathbf{A 1 9}$ could be obtained in about $93 \%$ yield.

For the selective reduction of the carboxylic of A19 in the presence of the nitrile groups, a suitable activation step was necessary (Scheme 5). Different methods for the activation of the carboxylic acid using reagents like chloroformates, cyanuric chloride or 1,1-carbonyldiimidazole were tested for the activation of the carboxylic acid, as were different reducing agents like sodium or potassium borohydrides for the reduction of the activated carboxylic acid

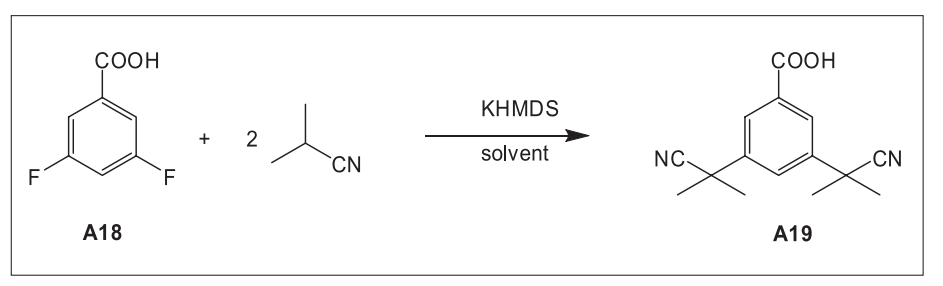

Scheme 4. Nucleophilic aromatic substitution of 3,5-difluoro-benzoic acid A18 with isobutyronitrile (KHMDS = potassium hexamethyldisilazide)

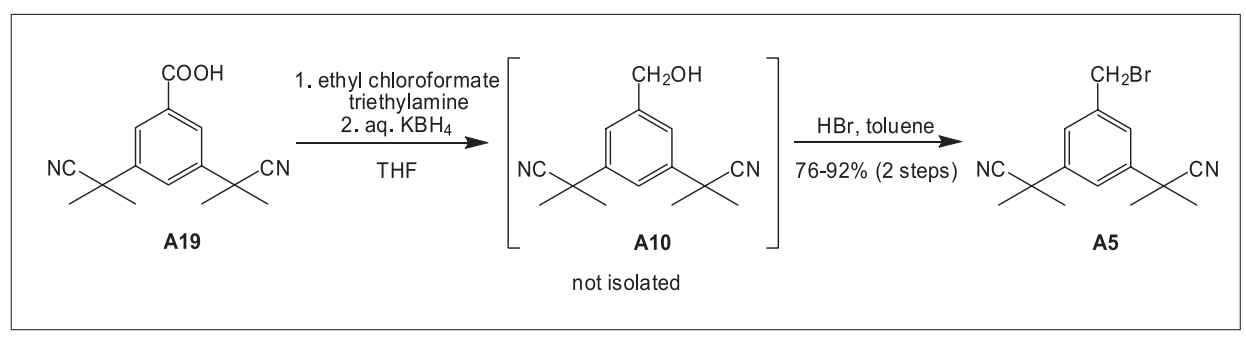

Scheme 5. Synthesis of 3,5-bis-(cyano-dimethyl-methyl)-benzylalcohol A5 from 3,5-bis-(cyanodimethyl-methyl)-benzoic acid A19 via intermediate A10

[13-15]. The best results so far have been obtained by reduction of the corresponding mixed anhydride prepared with isobutyl or ethyl chloroformate followed by reduction using potassium borohydride. Yields higher than $90 \%$ and excellent selectivities for the reduction step were obtained.

The high purity of compound A10 in the organic layer after an extractive work-up of the reaction mixture allowed us to use the crude extraction solution (toluene) directly for the next step without isolation of A10. Small amounts of residual starting material could be easily recovered from the aqueous extraction solvent.

Compound A5 was then synthesized by substitution of the benzylic alcohol using $48 \%$ aq. or $62 \%$ aq. $\mathrm{HBr}$ [16] with yields dependant on the reaction conditions. While using $62 \%$ aq. $\mathrm{HBr}$ accelerates the substitution, hydrolysis of the nitrile groups as side reaction is observed if the reaction is carried out at temperatures above room temperature. When $48 \%$ aq. $\mathrm{HBr}$ was used, the reaction proceeded at room temperature very slowly (ca. 40 h). However, almost quantitative yields could be obtained by performing the substitution with $48 \%$ aq. $\mathrm{HBr}$ at temperatures between $60{ }^{\circ} \mathrm{C}$ and $80^{\circ} \mathrm{C}$ for 1.5 to $2.5 \mathrm{~h}$. To avoid the simultaneous bromination of toluene, which gave the benzyl bromide as side product, the exclusion of light was necessary during the reaction.

The final step consists of the coupling reaction of benzyl bromide A5 with an appropriate triazole derivative. As previously mentioned, using e.g. triazole or sodium triazole for the final step, formation of the undesired regiosiomer A12 could not be avoided and resulted in loss of yield during its removal. Therefore a more selective reaction was required [17]. Two interesting routes were considered for the selective preparation of Anastrozole starting with the benzyl bromide A5 (Scheme 6). Route A begins with the reaction of compound $\mathbf{A 5}$ and sodium triazole which delivered a mixture of Anastrozole and its corresponding regiosiomer A12 in a ratio of about 88:12. However, it is known that 4-substituted 1,2,4-triazole such as e.g. 4-benzyl-1,2,4triazole can be isomerized into the thermodynamically more stable 1 -isomer at high temperatures in the presence of catalytic amount of the corresponding benzyl bromide [18]. Thus if a mixture of Anastrozole and its isomer A12 is treated in 1-methyl-2pyrrolidinone (NMP) with catalytic amount (0.1 equiv.) of the benzyl bromide $\mathbf{A 5}$ at temperatures of about $150{ }^{\circ} \mathrm{C}$, a complete conversion of compound A12 could be observed after $2 \mathrm{~h}$ at this temperature (determined by in-process HPLC analyses).

This reaction can be also carried out without prior isolation of the mixture of compounds A1 and A12. At first the alkylation of sodium triazole with 1.05 equiv. of benzyl bromide $\mathbf{A 5}$ in NMP gave at room temperature a mixture of $\mathbf{A 1}$ and $\mathbf{A 1 2}$ in a ratio of 91:9. After heating this mixture to $117{ }^{\circ} \mathrm{C}$ for $4 \mathrm{~h}$, an increased ratio of $98: 2$ was obtained.

A more elegant approach would be the selective alkylation of 1-substituted 1,2,4triazole derivatives bearing a suitable and easily removable protecting group. Using 1-trimethylsily-4H-1,2,4-triazole [19] for the preparation of Anastrozole, only trace amounts of the product was observed and both isomers were formed. Therefore this triazole derivative has no advantage. Another interesting triazole derivative is 4-amino-4H-1,2,4-triazole, which has previously been used as a precursor for the selective 


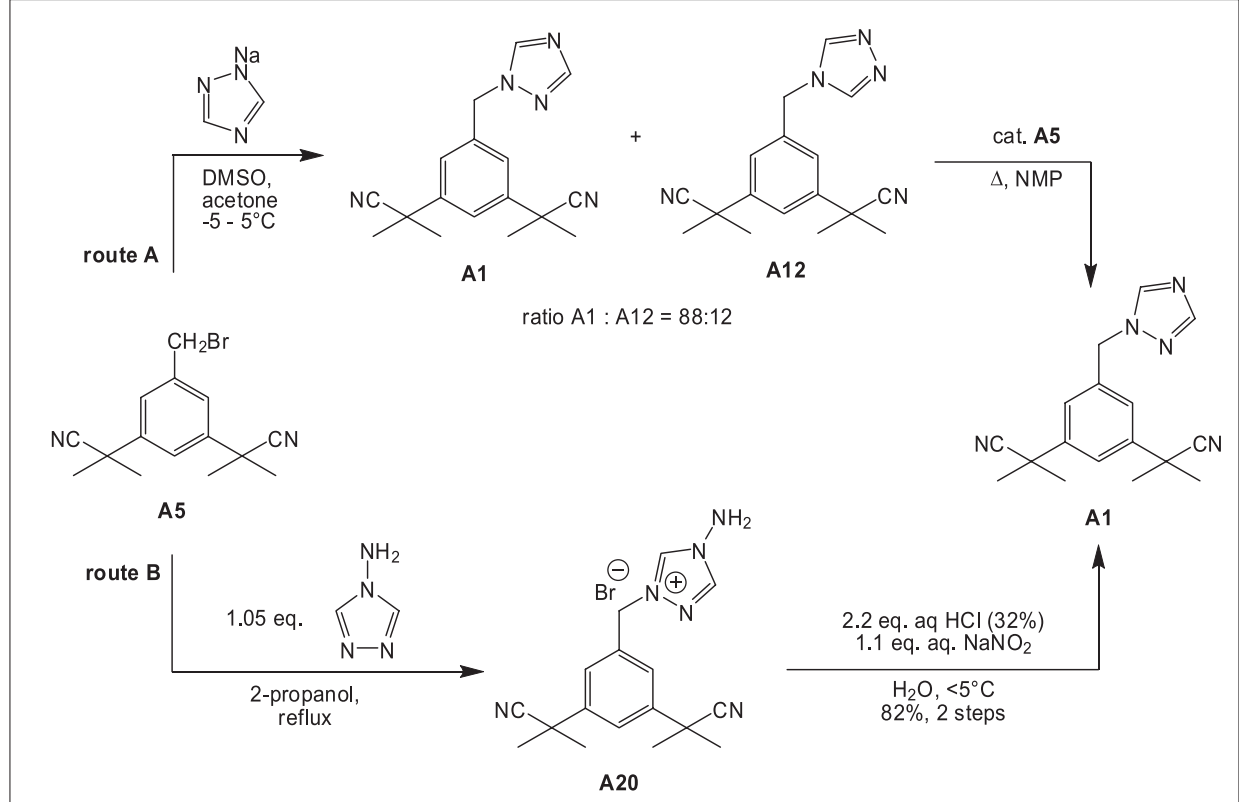

Scheme 6. Selective synthesis of Anastrozole A1 via route A by substitution of benzylic bromide A5 with sodium triazole followed by high temperature isomerization of A12 in the presence of A5 or alternatively via route B [20c] by selective alkylation of 4-amino-4H-1,2,4-triazole followed by deamination.

synthesis of 1-substituted 1,2,4-triazoles [17][20]. This method was also tested for the preparation of Anastrozole (route B [20c]). The reaction starts with the alkylation of 4amino-4H-1,2,4-triazole using benzyl bromide $\mathbf{A 5}$ to form the quaternary ammonium salt A20. Afterwards the amino-group could be removed by deamination with 1.1 equiv. sodium nitrite in the presence of hydrochloric acid. Anastrozole could be obtained in up to $82 \%$ yield (two steps) as a single isomer and in a purity of about $99.5 \%$ (HPLC) respectively $99.7 \%$ (GC) without any additional purification step. For further optimization, A1 may be prepared from A5 via $\mathbf{A 2 0}$ in a one-pot procedure.

In summary, an efficient and technically attractive new route for the selective preparation of Anastrozole has been found starting from readily available 3,5-difluorobenzoic acid and providing an overall yield of $58-70 \%$ obtained in a high purity $(\geq 99.5 \%$ by HPLC). The new process avoids the problematic separation of the desired product from its regiosiomer by fractional crystallization or column chromatography.

\subsection{Trandolapril}

\subsubsection{Introduction and Background}

In cooperation of the LPF with a contract partner, a development of an efficient scaleable route for the preparation of Trandolapril T1 (brand names: e.g. Gopten ${ }^{\circledR}$, Mavik $\left.^{\circledR}\right)$ was undertaken.

Trandolapril, $\{(2 S, 3 \mathrm{a} R, 7 \mathrm{a} S)-1-[[(2 S)-2$ -[[(1S)-1-carbethoxy-3-phenylpropyl] amino]-propanoyl]octahydro- $1 H$-indole2-carboxylic acid\} (Fig. 11), is the ethyl

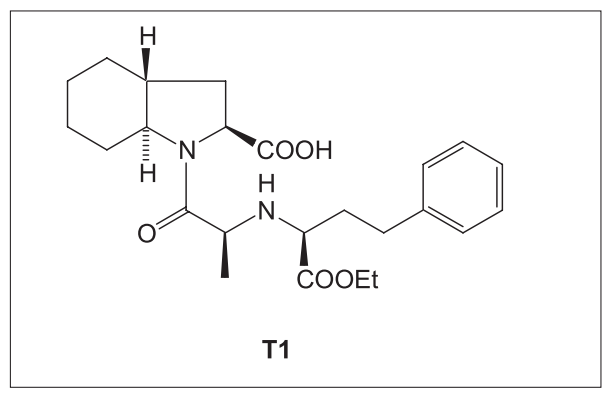

Fig. 11. Structure of Trandolapril

ester derivative of the diacid metabolite Trandolaprilat, an inhibitor of the peptidase 'Angiotensin Converting Enzyme' (ACE). Such inhibition of ACE activity results in an antihypertensive effect and therefore Trandolapril may be used to lower high blood pressure [21]. At present a number of strategies are known for the synthesis of Trandolapril. Most of the provided examples use the intermediates $\mathbf{T} 2$ or $\mathbf{T} 2 \mathbf{R}$ (trans-octahydroindole-2-carboxylic acid derivatives) and T3 or T3R (N-[1- $(S)$-ethoxycarbonyl3-phenylpropyl]-L-alanine-derivatives) (Scheme 7) which were coupled in the final step using typically activation methods e.g. DCC/HOBT, carboxylic halides, $\mathrm{N}$ sulfoxyanhydrides, N-carboxyanhydrides (Leuchs anhydride) to give Trandolapril T1 after removal of protecting groups, if present [22].

The first synthesis of Trandolapril [22a] was carried out by peptide coupling reaction of benzyl-protected racemic trans-octahydroindole-2-carboxylic acid (T2R, R = Bn) with N-[1-(S)-ethoxycarbonyl-3-phenylpropyl]-L-alanine $\mathbf{T 3}$ and $\mathrm{DCC} / \mathrm{HOBt}$ as coupling reagent. The diastereomeric mixture was separated by column chromatography whereupon Trandolapril could be obtained after removal of the protecting group. Further methods for the preparation of Trandolapril were published, but most of them used enantiopure trans-octahydroindole-2-carboxylic acid derivatives, which were prepared e.g. by esterification of the corresponding racemic acid and resolution of the ester $\mathbf{T} 2 \mathbf{R}$ ( $\mathrm{R}=$ protecting group) using suitable chiral acids [22h][22j][23]. The resolved trans-octahydroindole-2-carboxylic acid derivatives were then reacted with $\mathbf{T 3}$.

\subsubsection{Development of a New Route [22g]}

Our intention was to develop a simple, robust and scaleable method for the synthesis of Trandolapril which minimized the number of steps by avoiding the use of protection groups (Scheme 8). N-[1-(S)-ethoxycarbonyl-3-phenylpropyl]-L-alanine T3 is readily available in large amounts because of its extensive use as precursor for different ACE inhibitor syntheses [22f][24], and we decided to carry out the coupling with racemic trans-octahydroindole-2-carboxylic acid, $r a c-\mathbf{T} 2$, without prior resolution and without the use of protecting groups. For the activation of $\mathbf{T 3}$ we chose the Leuchs anhydride method, which activates the

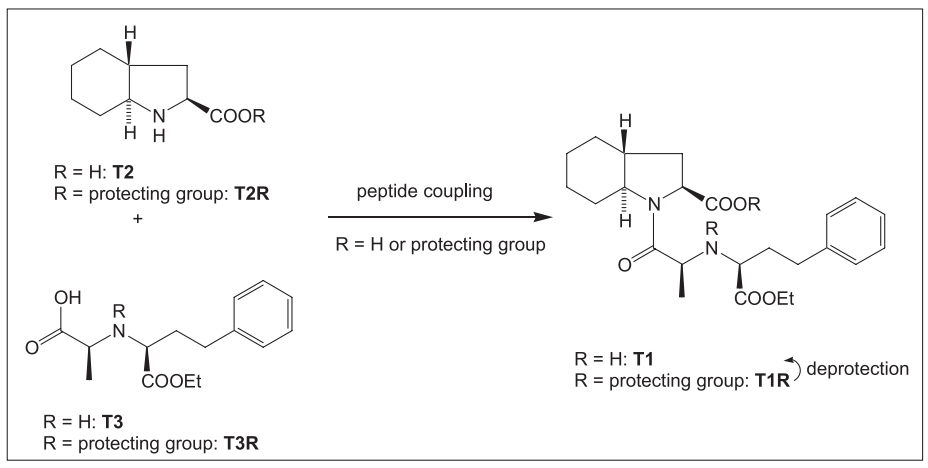

Scheme 7. Final coupling step for the synthesis of Trandolapril T1 using the precursors T2 or T2R and T3 or T3R 


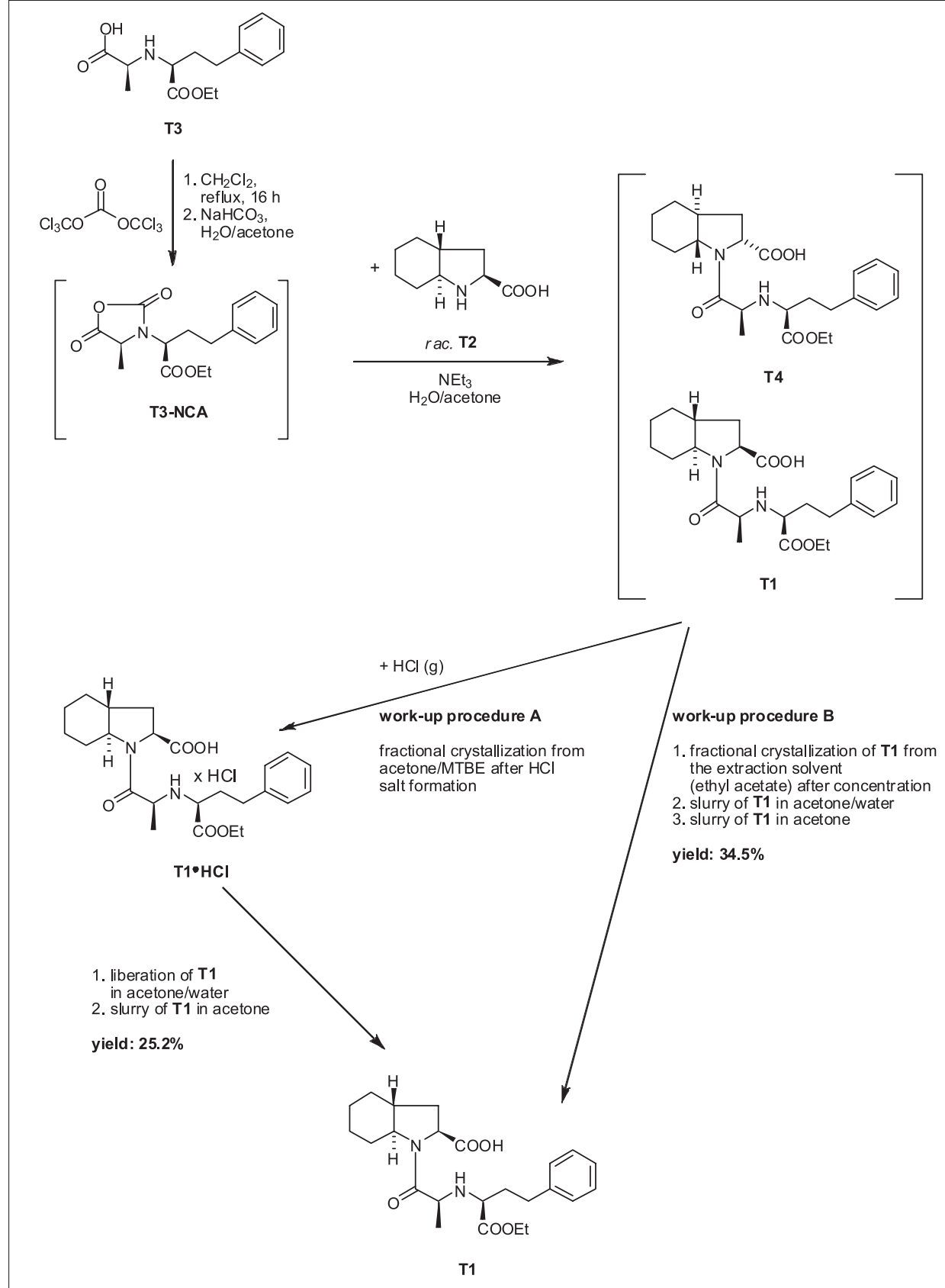

Scheme 8. Synthesis and isolation of Trandolapril. The reaction is carried out by coupling of the $\mathrm{N}$-carboxy-anhydride (NCA) of T3 (= T3-NCA) with racemic octahydroindole-carboxylic acid T2. The diastereomeric mixture consisting of $\mathbf{T} 1$ and $\mathbf{T 4}$ were separated by work-up procedure $\mathrm{A}$ using the corresponding $\mathrm{HCl}$ salts $\mathbf{T} 1 \cdot \mathbf{H C l}$ or alternatively by work-up procedure $\mathrm{B}$ without any prior salt formation.

carboxylic acid and protects the $\alpha$-amino group at the same time [22g][25].

Using the racemic mixture of $\mathbf{T} \mathbf{2}$ for the coupling step delivered Trandolapril T1 and its corresponding diastereomer T4. Two possible work-up procedures were developed to separate the diastereomeric mixture by fractional crystallization. The procedure A starts with $\mathrm{HCl}$ salt formation from a mixture of $\mathbf{T} \mathbf{1}$ and $\mathbf{T} \mathbf{4}$, obtained after extractive work-up of the reaction mixture using ethyl acetate. Exchange of the ethyl acetate by acetone followed by slow addition of MTBE initiates the selective crystallization of $\mathbf{T 1} \cdot \mathbf{H C l}$. Liberation of the free amine $\mathbf{T} 1$ from its $\mathrm{HCl}$ salt followed by slurrying of the final product first in a mixture of acetone and water and then in acetone gave Trandolapril in $25.2 \%$ yield and in a HPLC purity $\geq 99.9 \%$. As shown in Scheme 8 , the work-up procedure B proved to be more efficient since it avoids additional salt formation and solvent exchange for the fractional crystallization. The selective crystallization of $\mathbf{T} 1$ can be already carried out directly from the ethyl acetate used as extraction solvent. The crystallization was initiated by concentration of the organic layer. Additional slurrying of the crude product in a mixture of acetone/water and then in acetone gave the final product in $34.5 \%$ yield and in a HPLC purity $\geq 99.9 \%$. In both cases the final slurry in acetone removes not only residual impurities and water but also ensures the formation of the desired pure and stable crystalline form.

Because ref. [22a] does not provide any data for crystallization of Trandolapril and investigations of crystalline forms were published only relatively recently [22c], it was necessary to identify the possible crystalline forms of Trandolapril. Initial crystallization experiments led to the identification of two crystalline forms, referred to here as Form A and Form B. Form A was obtained e.g. after the above-described work-up procedures, while Form B could be obtained by adding a solution of Trandolapril in methanol or acetone to a five-fold excess water at $0-5{ }^{\circ} \mathrm{C}$. Determination of the water content of crystalline Form B (4.0-4.4\%) after drying indicated the presence of a monohydrate and therefore a pseudopolymorphic form of Trandolapril.

Exhaustive polymorph screening by e.g. solvent variation, investigation of different cooling rates, evaporation, slurrying or precipitation at different temperatures in different solvent systems, resulted in the identification of a less stable polymorphic Form C and an amorphous Form [26]. Typical XRD measurements of these forms are shown in Fig. 12.

In summary, an efficient and scaleable method for the preparation of highly pure Trandolapril was developed. After transfer of the technology to a contract manufacturer, the production of Trandolapril was successfully validated.

\section{Conclusion and Outlook}

With the foundation of the LPF as a joint venture between the University of Zurich and the pharmaceutical industry, a unique model has been established for how such cooperation can work to the benefit of partners, clients and employees. On the one hand the LPF incorporates highly motivated and well-trained staff with university and vocational backgrounds working in a state-of-the-art equipped environment that is uncommon for an academic institution. Process chemistry can be performed at the highest level, comprising all steps necessary for the development of projects from bench to production, under GMP compliance. Within the last decade, a large number of projects were successfully developed, in which many contain new innovative routes or steps resulting in several patents. Customers and clients could always rely on a full range of services, from patent research to full development of the projects, and including 


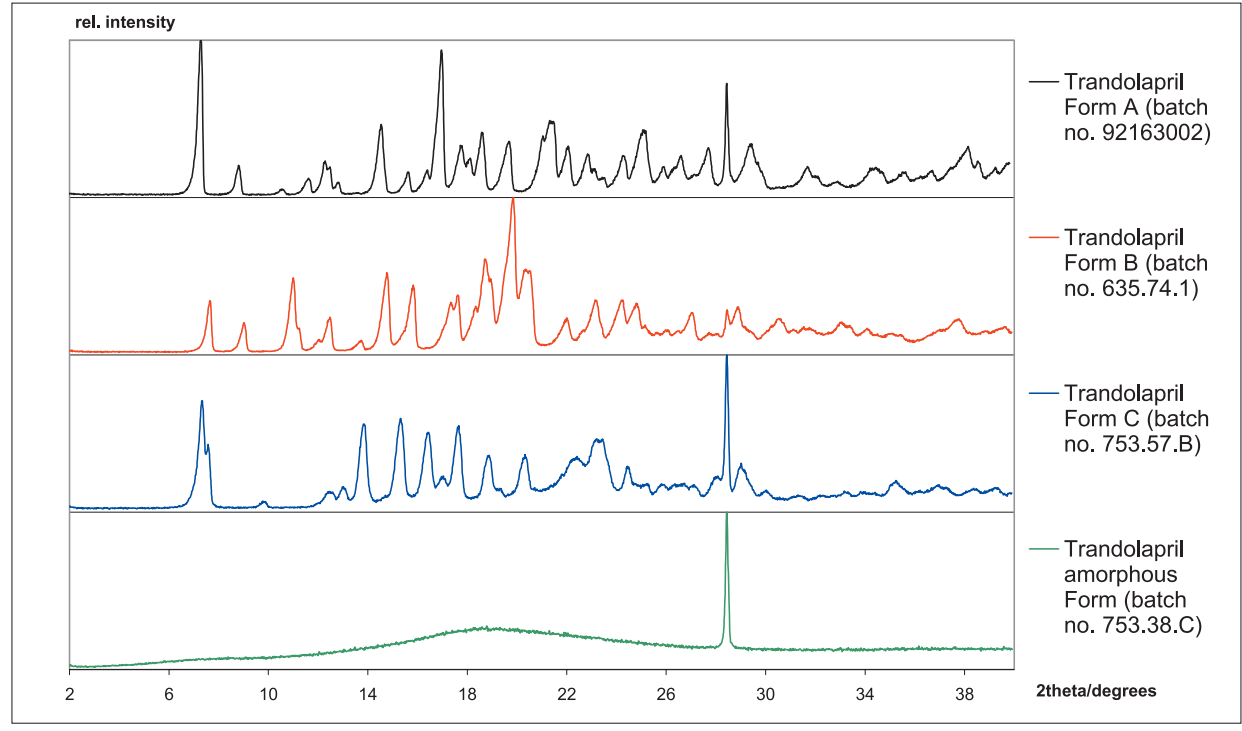

Fig. 12. Diffractograms of Trandolapril crystalline and amorphous forms. All XRD measurements were measured in the presence of silicon as internal standard.

the whole palette of analytics. On the other hand the LPF offers a training platform for postdocs in a discipline that is not normally part of their academic education. The real life training in how to develop a chemical project according to current industrial standards and under commercial pressure makes them better prepared and more attractive for future job positions.

\section{Acknowledgements}

Special gratitude has to be expressed to the University of Zurich, Cilag AG and AZAD Pharma AG for founding, creating and supporting the Laboratory for Process Research as a unique model for performing and learning process chemistry in an academic environment. I would like to thank Professor Dr. Jay S. Siegel and all former and current LPF members for the always successful work and cooperation. For the careful review of this article I would like to thank Prof. Dr. Nathaniel Finney.

Received: June 27, 2006

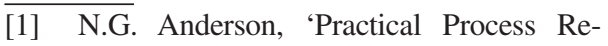
search \& Development', Academic Press, San Diego, 2000.

[2] a) P.V.Plourde, M.Dyroff, M.Dukes, Breast Cancer Res. Treat. 1994, 30, 103-111; b) R.A. Yates, M. Dowsett, G.V. Fisher, A. Selen, P.J. Wyld, Br. J. Cancer 1996, 73, 543-548; c) J. Geisler, N. King, M. Dowsett, L. Ottestad, S. Lundgren, P. Walton, P.O. Kormeset, P.E. Lonning, Br. J. Cancer 1996, 74, 1286-1291; d) P.V. Plourde, M. Dyroff, M. Dowsett, L. Demers, R. Yares, A. Webster, J. Steroid. Biochem. Molec. Biol. 1995, 53, 175-179; e) M. Dukes, P.N. Edwards, M. Large, I.K. Smith, T. Boyle, J. Steroid. Biochem. Molec. Biol. 1996, 58 , 439-445; e) R.W. Brueggemeier, J.C. Hackett, E.S. Diaz-Cruz, Endocrine Reviews 2005, 26, 331-345.

[3] P.N. Edwards, M.S. Large, EP Patent No. 02967491 B1, 1994.
[4] P. Liu, Y. Chen, J. Deng, Y. Tu, Synthesis 2001, 14, 2078-2080.

[5] M. Engel, C.W. Burris, C.A. Slate, B.W. Erickson, Tetrahedron 1993, 49, 87618770.

[6] UK Chemical Reaction Hazards Forum, incident101, 'Sodium Hydride / DMF process stopped', http://www.crhf.org.uk.

[7] M. Pulla Reddy, N. Venkaiah Chowdary, WO Patent No. 2005105762 A1, 2005.

[8] M. Alnabari, B. Freger, O. Arad, L Zelikovitch, Y. Servi, E. Danon, G. Davidi, J.Kaspi, US Patent No. 20060035950 A1, 2006.

[9] A. Gaitonide, C. Vaidya, S.R. Pawar, WO Patent No. 2006000836, 2006.

[10] M. Sundermeier, L. Ulmer, T. Bader, Laboratory for Process Research, University Zurich (Switzerland), manuscript in preparation.

[11] D.A. Culkin, J.F. Hartwig, J. Am. Chem. Soc. 2002, 124, 9330-9331.

[12] a) S. Caron, E. Vazquez, J.M. Wojcik, $J$. Am. Chem. Soc. 2000, 122, 712-713; b) S. Caron, EP Patent No. 1046635 B1, 2004.

[13] a) K. Soai, S. Yokoyama, K. Mochida, Synthesis 1987, 647-648; b) G. Kokotos, Synthesis 1990, 299-301; c) T. Itaya, T. Fujii, A. Evidente, G. Randazzo, G. Surcio, N.S. Iacobellis, Tetrahedron Lett. 1986, 27, 63496352; d) M. Rodriguez, M. Llinares, S. Doulut, A. Heitz, J. Martinez, Tetrahedron Lett. 1991, 32, 923-926; e) E.B. Fray, C.J. Moody, P. Shah, Tetrahedron 1993, 49, 439-450.

[14] M. Falorni, A. Porcheddu, M. Taddei, Tetrahedron Lett. 1999, 40, 4395-4396.

[15] a) A.A. Cordi, P. Desos, E. Ruano, H. Al-Badri, C. Fugier, A.G. Chapman, B.S. Meldrum, J.-Y. Thomas, A. Roger, P. Lestage, Farmaco 2002, 57, 787-802; b) H.A. Staab, Angew. Chem., Int. Ed. Engl. 1962, 1, 351-367; c) Y.G. Perron, L.B. Crast, J.M. Essery, R.R. Fraser, C.J. Godfrey, C.T. Holdrege, W.F. Minor, M.E. Neubert, R.A. Partyka, L.C.Cheney, J. Med. Chem. 1964, 7, 483-487.
[16] a) F. Vögtle, N. Eisen, S. Franken, P. Büllesbach, H. Puff, J. Org. Chem. 1987, 52, 5560-5564; b) K.M. Doxsee, M. Feigel, K.D. Stewart, J.W. Canary, C.B. Knobler, D.J. Cram, J. Am. Chem. Soc. 1987, 109, 3098-3107.

[17] M. Balasubramanian, J.G. Keay, E.F.V Scriven, Heterocycles 1994, 37, 19511975.

[18] a) R.M. Claramunt, J. Elguero, R. Garceran, Heterocycles 1985, 23, 2895-2906; b) K. Smith, A. Small, M.G. Hutchings, Chem. Lett. 1990, 347-350.

[19] J.P. Gasaparini, R. Gassend, J.C. Maire, J. Elguero, J. Organomet. Chem. 1980, 188, 141-150.

[20] a) B.A. Astleford, G.L. Goe, J.G. Keay, E.F.V. Scriven, J. Org. Chem. 1989, 54, 731-732; b) H.V. Patel, R.J. Jani, T. Thennati, WO Patent No. 2004076409, 2004; c) B.L. Edgar, F.R. Zuleski, J.R. Harding, 'Synthesis of carbon-14 labelled forms of anastrozole', Synthesis and Applications of Isotopically Labelled Compounds, Proceedings of the International Symposium, 7th, Dresden, Germany June 18-22, 2000, 2001, 178-180.

[21] D.C. Peters, S. Noble, G.L. Plosker, Drugs 1998, 56, 871-893.

[22] a) H. Urbach, R. Henning, V. Teetz, R. Geiger, R. Becker, H. Gaul, EP Patent No. 0084164 B1, 1987; b) S. Serra Mortes, A. Palomo Coll, R. Zupet, WO Patent No. 9633984, 1996; c) R.B. Parthasaradhi, R.K. Rathnakar, R.R. Raji, R.B. Narasa, R.D. Muralidhara, WO Patent No. 2004076417 , 2004; d) H. Shimamura, JP Patent No. 2004307340, 2004; e) B. Jenko, WO Patent No. 2004101515, 2004; f) G.P. Singh, H.M. Godbole, P.R. Mahajan, S.P. Nehate, WO Patent No. 2005010028, 2005; g) M. Pogutter, F. Rudolf, H.-U. Bichsel, T. Bader, WO Patent No. 2005051909, 2005; h) P. Cid, WO Patent No. 2005054194, 2005; i) N. S. Joshi, S.B. Bhirud, B.P. Ramam, A.R. Bodkhe, US Patent No. 20060079698 A1, 2006; j) P.P. Reddy, S.S. Chitre, S. Polavarapu, V. Vakamudi Sri Naga Venkata Laxmi, WO Patent No. 2006014916, 2006.

[23] H. Shimamura, Y. Nakata, WO Patent No. $2004065368,2004$.

[24] a) M. Kottenhahn, H. Harr, K. Drauz, EP Patent No. 0523449 B, 1996; b) A. Matsumoto, M. Nomura, Y. Kogame, Y. Ueda, WO Patent No. 02074728, 2002; c) K.A. Hackl, EP Patent No. 0789029 B1, 2003.

[25] a) J.W. Skiles, R.D. Youssefyeh, J.T. Suh, H. Jones EP Patent No. 0061768 B1, 1986; b) F.-C. Huang, H. Jones, C.J. Lin, B. Love, EP Patent No. 0114067, 1987; c) S. Takahashi, K. Inoue, Y. Yanagida, T. Ohashi, K. Watanabe, EP Patent No. 0215335 B1, 1992; d) C.-M. Chen, Y.-L. Liu, Y.-C. Chao, C.-H. Wu, EP Patent No. 1197490 B1, 2004.

[26] L. Ulmer, Laboratory for Process Research, University Zurich (Switzerland), unpublished results. 